\title{
Forward charm-production models and prompt neutrinos at IceCube
}

\section{Atri Bhattacharya and J.R. Cudell}

Space sciences and Technologies for Astrophysics Research (STAR) Institute, University of Liège, Liège, Belgium

E-mail: A.Bhattacharya@uliege.be, JR.Cudell@uliege.be

ABSTRACT: We investigate the prompt neutrino background at IceCube, as determined from forward charm. We consider the role of intrinsic charm and of a recombination model and show that the contribution of these mechanisms is at most a factor two.

KeYwords: Neutrino Physics, Solar and Atmospheric Neutrinos

ARXIV EPRINT: 1808.00293 


\section{Contents}

1 High-rapidity D-meson from the high- $x_{F}$ NLO contribution 2

2 Intrinsic charm 3

3 The BJM recombination formalism $\quad 5$

4 Prompt neutrino fluxes from the best-fit cross-section 6

$\begin{array}{lll}5 & \text { Event rates } & 8\end{array}$

$\begin{array}{llr}6 & \text { Conclusions } & 9\end{array}$

\section{Introduction}

This century is witnessing the birth of a new astronomy making use of all the fundamental interactions to explore hidden processes in the Universe: the multi-messenger approach which enjoyed its first successes with the observation of GW170817/GRB170817A [1], and with the recent IceCube breakthrough [2] which identified the blazar TXS 0506+056 as the source of IceCube-170922A. This method will facilitate easier characterisation of sources, as well as provide a new tool to discover unknown objects [3]. It may also open a window to particle physics discoveries $[4,5]$. Important players in this multi-messenger program are the neutrino telescopes IceCube and ANTARES/KM3NET, which use large volumes of ice/water in the Earth. Their main drawback is that the Earth comes with an atmosphere, which produces significant background from the collisions of cosmic rays on the air. These collisions, via the weak decay of light mesons, produce a large neutrino background. However, at high energy, these light mesons interact with air molecules before having the chance to decay. Hence, the neutrino background is increasingly suppressed with higher energies and higher time dilation. However, there is one exception to this mesons containing heavy quarks will quickly decay and produce neutrinos. Heavier quarks are produced through more energetic gluons, which are scarce compared to low-energy ones. This means that $b \bar{b}$ and $t \bar{t}$ production is suppressed compared to $c \bar{c}$. Consequently, the main background at high energies comes from decays of charmed mesons.

The highest-energy neutrino background comes from forward production, and this implies that the charm mass is the only available scale for perturbative QCD. Inclusive QCD calculations of quark production start to make sense at the charm mass scale but require at least a next-to-leading order evaluation [6-9]. However, we are interested in the semi-inclusive calculation as the charm quark is singled out at the high momentum, and this implies we are sensitive to non-perturbative effects which cannot be factored out into fragmentation or structure functions. This makes the highest-energy charm background 
model dependent. Recent perturbative calculations of charmed-meson production are already consistent with observations from the latest collider experiments, including ALICE, ATLAS, and CMS at low rapidities ${ }^{1}$ This only leaves a small window of rapidities where the diffractive cross-section can significantly contribute, viz. at very high rapidities.

There are currently no data corresponding to $D^{ \pm, 0}$ production at rapidities beyond those accessible to $\mathrm{LHCb}$ at $\sqrt{s}=13 \mathrm{TeV}$; as a consequence the constraints on theoretical parameters governing the forward production of these mesons are rather weak. At these very high rapidities, two classes of models are usually considered to describe the production of heavy mesons: a) models where the charm can intrinsically carry a large momentum that can then be inherited by the D mesons [11-13], and $b$ ) those that assume that the $\mathrm{D}$ is boosted because its light quark is a spectator valence quark from one of the initial nucleons [14-17]. The former is straightforwardly implemented via modified structure functions, whereas the latter require dedicated computations involving new fragmentation mechanisms. Our goal in this work is to estimate the maximum prompt atmospheric neutrino flux that may be seen at IceCube by allowing the parameters in these models to go as high as possible whilst still maintaining consistency with data at central rapidities.

This paper is organised as follows: in section 1, we discuss the predictions of the leading-twist NLO charm production calculation for very high rapidities, and the corresponding uncertainties involved. In section 2, we consider and constrain the possible modification to the charm momentum from intrinsic charm [11]. In section 3, we consider the recombination process of Braaten, Jia and Mehen [14-16]. Finally, in section 4, we estimate the corresponding prompt neutrino fluxes, and show that this component of the background remains small in all cases.

\section{High-rapidity D-meson from the high- $x_{F}$ NLO contribution}

As previously noted, the perturbative treatment of Bhattacharya-Enberg-Reno-SarcevicStasto (BERSS) $[8,9]$ has been tuned to the experimental results for D-meson production cross-sections across a wide range of energies. At the low-energy end of the spectrum, this includes data from fixed-target, proton and pion beam experiments at the CERN SPS at a few hundred $\mathrm{GeV}$, and the $920 \mathrm{GeV}$ fixed-target experiment HERA-B; further up the energy ladder are the somewhat discordant results from STAR and PHENIX fixed-target experiments, both involving a beam of energy $200 \mathrm{TeV}$; and finally, constraints at the highest energies come from D-meson production data at the LHC at $\sqrt{s}=7$ and $13 \mathrm{TeV}$. The total number of experimental points is thirteen.

The tunable parameters in the theory are the charm mass and the factorisation and renormalisation scales. By fitting the theoretical cross-sections, computed perturbatively to the next-to-leading order, to observed data at these widely disparate energies, one is able to obtain the best-fit and limiting values of these scales consistent with observations. The overall fit has a $\chi^{2} /$ d.o.f. of 1.31. Allowing the factorisation and renormalisation scales to

\footnotetext{
${ }^{1}$ The LHCb data, at central rapidities, are well reproduced at $\sqrt{s}=5 \mathrm{TeV}$ and $7 \mathrm{TeV}$. At $13 \mathrm{TeV}$, it may undershoot the prediction although one should note that the data have been revised twice and the preprint five times [10]. If this result is confirmed, it will only strengthen our conclusion.
} 

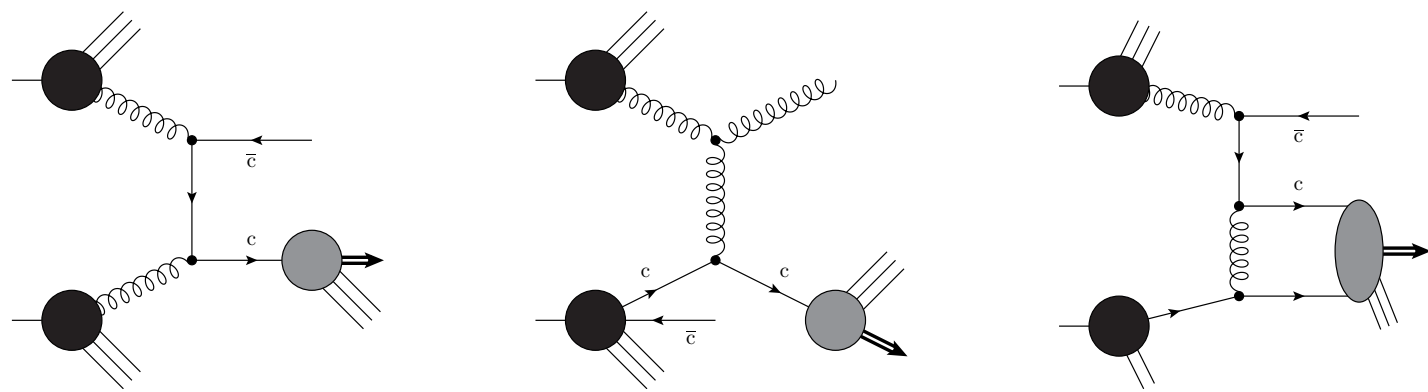

Figure 1. Different processes leading to forward-charmed-meson production: (from left to right) perturbative gluon fusion in the vein of BERSS followed by fragmentation, intrinsic-charm-initiated $D$-meson production followed by fragmentation, and charm-pair production leading to $D$-mesons via recombination.

run proportionally to the transverse mass of the charm quark $m_{T}=\sqrt{m_{c}^{2}+p_{T}^{2}}$, where $p_{T}$ is its transverse momentum, the BERSS analysis finds best fit values at $\left(M_{F} / m_{T}, \mu_{R} / m_{T}\right)=$ $(2.1,1.6)$ for the choice of fixed $m_{c}=1.27 \mathrm{GeV}$. The upper and lower limits, determined with reference to $1 \sigma$ errors on experimental data, are shown in [8].

\section{Intrinsic charm}

The simplest way to boost charm is to recognise that sometimes the proton has a fluctuation that produces a $c \bar{c}$ pair, as shown in figure 1. This was recognised a long time ago [11] and lead to the introduction of intrinsic charm. It is the part of the structure functions that comes from the non-perturbative large-distance gluon field and it cannot be described via DGLAP evolution. Modern structure functions include this contribution as an initial parametrisation in some of their sets. Here we will consider the latest parametrisation due to the CT collaboration [18]. They have looked at the intrinsic charm content in the proton at the leading (LO), next-to-leading (NLO) and next-to-next-to-leading orders (NNLO). The structure functions are fit to the open-charm production data in the central rapidity region from recent collider experiments. The CT14 sets propose four distinct parametrisations of the intrinsic charm distribution function (shown in figure 2), and we choose the 'BHPS2' set, which has the highest charm content at large $x$.

As the contribution of intrinsic charm will turn out to be small, it is sufficient to look at the leading-order calculation, for which we use the expressions of [13]. There is a large uncertainty attached to the value of the quark mass, and we choose here a small charm mass, $m_{c}=1.27 \mathrm{GeV}$, that corresponds to a high neutrino flux, as in $[4,5]$. We also use leading-order structure functions, and keep the Kramer-Kniehl fragmentation functions [19]. The results are shown in figure 3 , for several representative values of $\sqrt{s}$, where we add the intrinsic charm contribution to the NLO gluon-fusion cross section. We see that intrinsic charm makes a difference only at very high $x_{F}>0.9$ where it can change the differential cross section by a factor of two of larger. However, this is the region of $x_{F}$ in which the differential cross section is suppressed, and we shall see that the effect on 


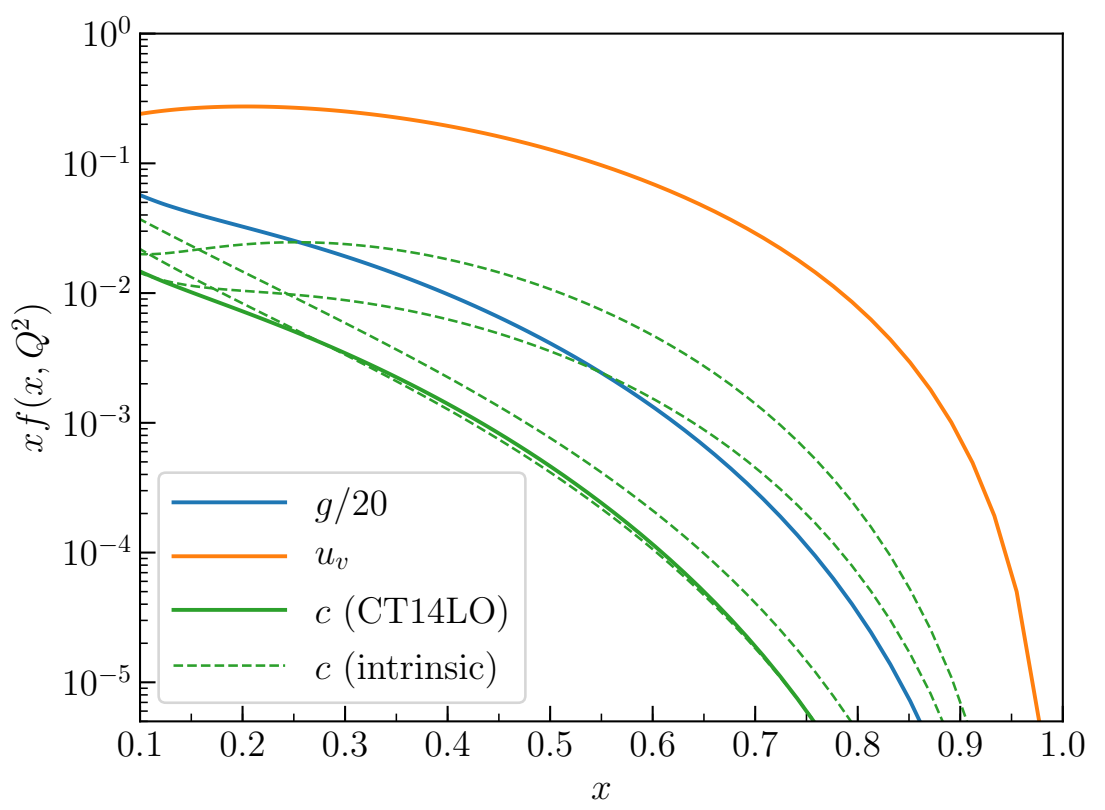

Figure 2. Leading-order CT14 parton distributions for gluons, valence up quarks and charm quarks at $Q^{2}=4 m_{c}^{2}$. Also shown (dashed green curves) are the four intrinsic charm parametrisations as part of the CT14LO-IC set.

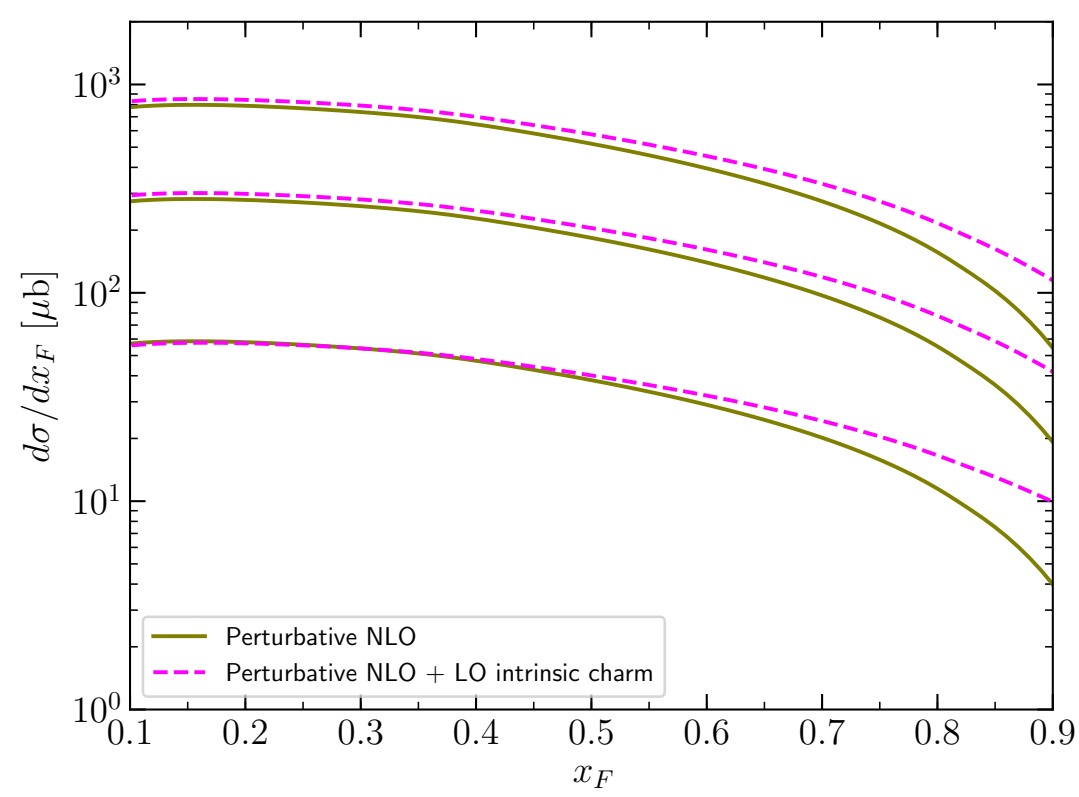

Figure 3. Total D-meson production cross section $d \sigma / d x_{F}$ as a function of the final-state meson $x_{F}$ without and with intrinsic charm at $\sqrt{s}=10^{2}, 10^{3}$, and $10^{4} \mathrm{GeV}$ from bottom to top. 
the neutrino flux is small. ${ }^{2}$ Our prediction for the prompt neutrino flux when including intrinsic charm contribution is consistent with results from other recent works [21-23]. It is significantly below the intrinsic charm contribution to the flux discussed in [24], which uses $\Lambda^{0, \pm}$ production data from fixed-target experiments to deduce constraints on $D^{0, \pm}$ production assuming extreme values of fractional momentum transferred to the final $\bar{c} c$ pair. As such, the flux derived therein must be seen as a weak upper limit to the intrinsic charm contribution and our results are well within this limit. Note also that the NLO calculation is infrared finite and includes the mechanism considered in [25] without the need for an infrared cut-off.

\section{The BJM recombination formalism}

In the Braaten-Jia-Mehen (BJM) formalism for heavy-quark recombination with an active light quark, the $D$-production cross section is expressed to leading order as product of two factors:

$$
d \hat{\sigma}[D]=d \hat{\sigma}[\bar{q} g \rightarrow[\bar{q} c(n)]+\bar{c}] \times \rho[[\bar{q} c(n)] \rightarrow D] .
$$

The first factor is the usual perturbative term. Whereas the standard calculation (BERSS) calculates this factor to the next-to-leading order and then multiplies it by fragmentation functions to make $D$-mesons, an extra contribution is considered here, in which the light quarks do not come from the vacuum but rather from the proton. This contribution is parametrised by the non-perturbative numerical factor $\rho[[\bar{q} c(n)] \rightarrow D]$, which is calculated from an effective Lagrangian. The quantity $n$ is representative of the colour and spin quantum numbers of the $\bar{q} c$ pair. This formalism allows for coloured bound states or spinflips. For example, the production of a colour singlet, spin-conserving $D^{+}$meson is given by the product of

$$
\begin{aligned}
\frac{d \hat{\sigma}}{d t}\left[\bar{d} c\left({ }^{1} S_{0}^{(1)}\right)\right]= & \frac{2 \pi^{2} \alpha_{s}^{3}}{243} \frac{m_{c}^{2}}{S^{3}}\left[-\frac{64 U}{S}+\frac{m_{c}^{2}}{T}\left(79-\frac{112 U}{T}-\frac{64 U^{2}}{T^{2}}\right)\right. \\
& \left.-\frac{16 m_{c}^{4} S}{U T^{2}}\left(1-\frac{8 U}{T}\right)\right]
\end{aligned}
$$

and $\rho_{1}^{\mathrm{sm}}=\rho\left[\bar{d} c\left({ }^{1} S_{0}^{(1)}\right) \rightarrow D^{+}\right]$, where, "sm" indicates a spin-matched pair, i.e. $c$ and $\bar{d}$ have matching spins, and $S, T$, and $U$ are modified Mandelstam variables that can be expressed in terms of the final state transverse momentum $p_{\perp}$ and rapidity $\Delta y$ as

$$
\begin{aligned}
& S=2\left(p_{\perp}^{2}+m_{c}^{2}\right)(1+\cosh \Delta y), \\
& T=-\left(p_{\perp}^{2}+m_{c}^{2}\right)\left(1+e^{-\Delta y}\right), \\
& U=-\left(p_{\perp}^{2}+m_{c}^{2}\right)\left(1+e^{\Delta y}\right) .
\end{aligned}
$$

Expressions for other cross-sections are given in $[14,15]$. Note that if one includes fragmentations $\left[\bar{d} c\left({ }^{1} S_{0}^{(1)}\right) \rightarrow D^{+} X\right]$ then $\rho$ becomes a free positive parameter, that can be

\footnotetext{
${ }^{2}$ This is even before taking into account the kinematic suppression of the charm pair production crosssection from intrinsic charm as discussed in ref. [20]. This would appreciably reduce its contribution to the neutrino flux.
} 

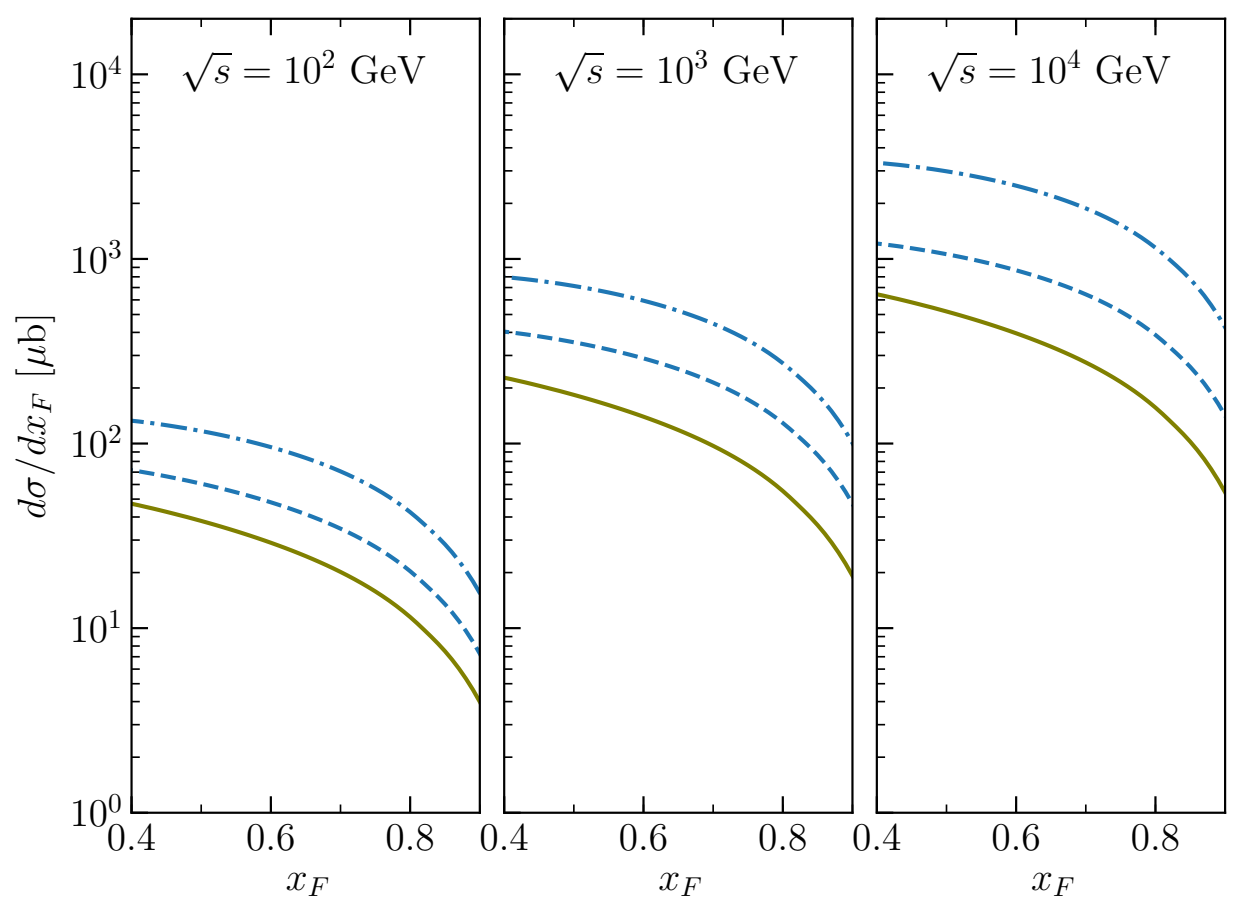

Figure 4. Total $D$-meson production cross section $d \sigma / d x_{F}$ as a function of the final-state meson $x_{F}$. The NLO perturbative calculation is shown as the green, solid curve and the differential crosssection when including the BJM model with $\rho=0.06(0.15)$ is shown as dashed (dot-dashed), blue curve for $\sqrt{s}=10^{2}, 10^{3}$, and $10^{4} \mathrm{GeV}$.

adjusted to the data. BJM found that one only needs the colour-singlet spin-conserving term to reproduce the high- $x_{F}$ charge asymetries in $D^{+} / D^{-}$production observed in $\pi^{-} p$ by the E791 experiment, with a value $\rho_{1}=0.06$. They also fitted photoproduction data, which leads to $\rho_{1} \approx 0.15$. More recently, these expressions have been used [26] to reproduce the $D$ asymmetry observed at LHCb. There it was found, at somewhat lower values of $x_{F}$, that coulour-singlet spin-non-flip terms were not enough to reproduce the asymmetry. As we concentrate on the high- $x_{F}$ data, we shall adopt the BJM result, and consider the interval $[0.06,0.15]$ for $\rho$.

We see in figure 4 that the inclusion of the recombination enhances the forward $D^{ \pm, 0}$ production by up to a factor of 2 for the higher value of $\rho=0.15$ at $x_{F} \approx 0.9$. Note that the BJM model is valid at high $x_{F}$ only and that it may get substantial corrections at low and medium values of $x_{F}$, hence the beginning of the curve of figure 4 is only indicative.

\section{Prompt neutrino fluxes from the best-fit cross-section}

Using the BJM cross-sections with the best-fit parameters above, we can determine the corresponding neutrino flux expected at Earth as a consequence of interactions of cosmic rays with atmospheric nuclei assumed to contain $A$ nucleons. As an estimate of the incident cosmic-ray proton flux, we use the models of Gaisser [27], specifically his proton-rich esti- 


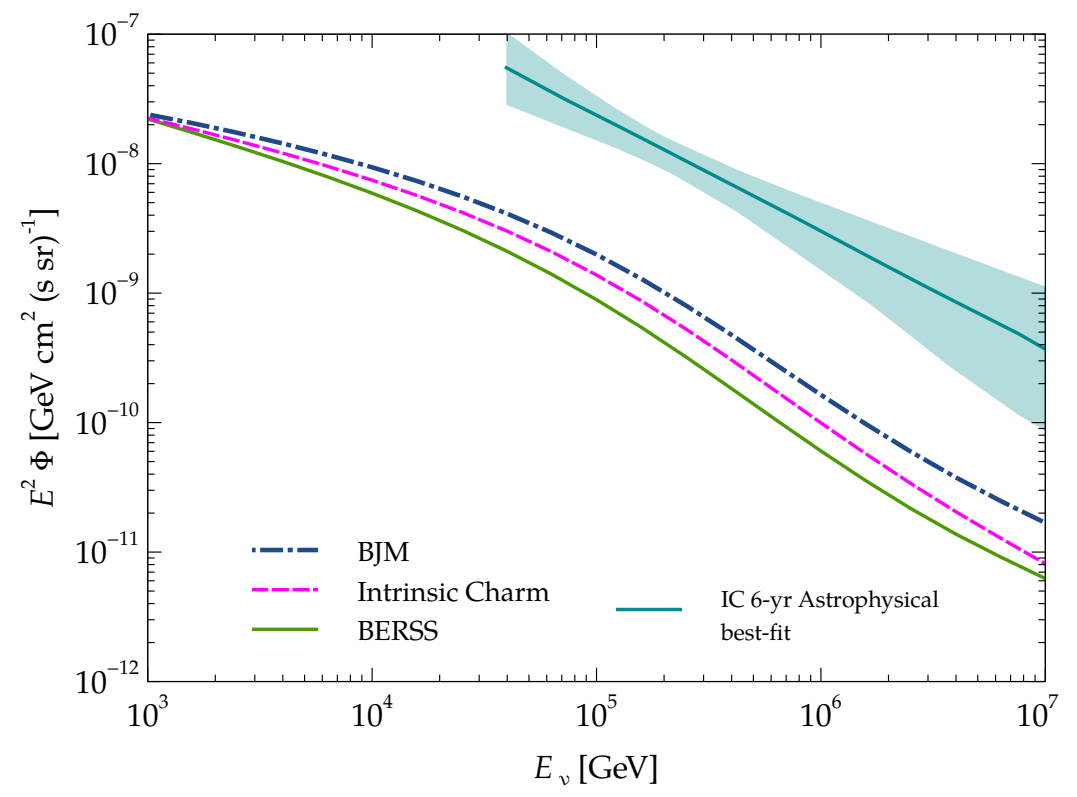

Figure 5. The results for prompt fluxes when including forward charm production from different formulations.

mates designated as $H 3 p$. The computation of the corresponding neutrino flux follows the semi-analytical procedure outlined in standard literature (see e.g., [28, 29]). Briefly, working in the exponential atmosphere approximation, where the column density as a function of height is given by $\rho(h)=\rho_{0} \exp \left(h / h_{0}\right)$ with $\rho_{0}=2.03 \times 10^{-3} \mathrm{~g} / \mathrm{cm}^{3}$ and $h_{0}=6.4 \mathrm{~km}$, the low- and high-energy lepton fluxes may be expressed in simple semi-analytic forms in terms of spectrum-weighted $Z$-moments. These $Z$-moments relate to the conversion of the incoming proton content in the cosmic-ray flux to the heavy-meson flux produced therefrom $\left(Z_{p h}\right)$, and then from the latter to the final leptonic flux reaching the detector $\left(Z_{h \ell}\right)$. Additional moments $Z_{p p}$ and $Z_{h h}$ describe respectively the energy losses of the protons in collisions with air nuclei not leading to meson production, and the energy losses of the meson before their decay resulting in leptons. The full procedure is described in [8], and, for brevity, we refer the reader to the discussion in section 3 thereof rather than repeat it here. Using this machinery, but with additional contributions to $D^{ \pm, 0}$ production from non-perturbative diffractive processes in the forward $x_{F}$ region, we have computed the total prompt neutrino flux in the intrinsic-charm and scenarios. These are shown in figure 5.

Note that the NLO amplitude does not interfere with the BJM amplitude given that they fragment differently and, hence, the two final states are different. In the intrinsic charm case, the kinematics of the charm quarks prevents significant interference. As far as BJM and intrinsic charm go, the cross-sections could in principle be added; however, the fits with intrinsic charm structure functions do not include the BJM mechanism and these two processes are, therefore, not independent. The inclusion of the BJM mechanism in the fits would lower the contribution of intrinsic charm at high $x_{F}$. 


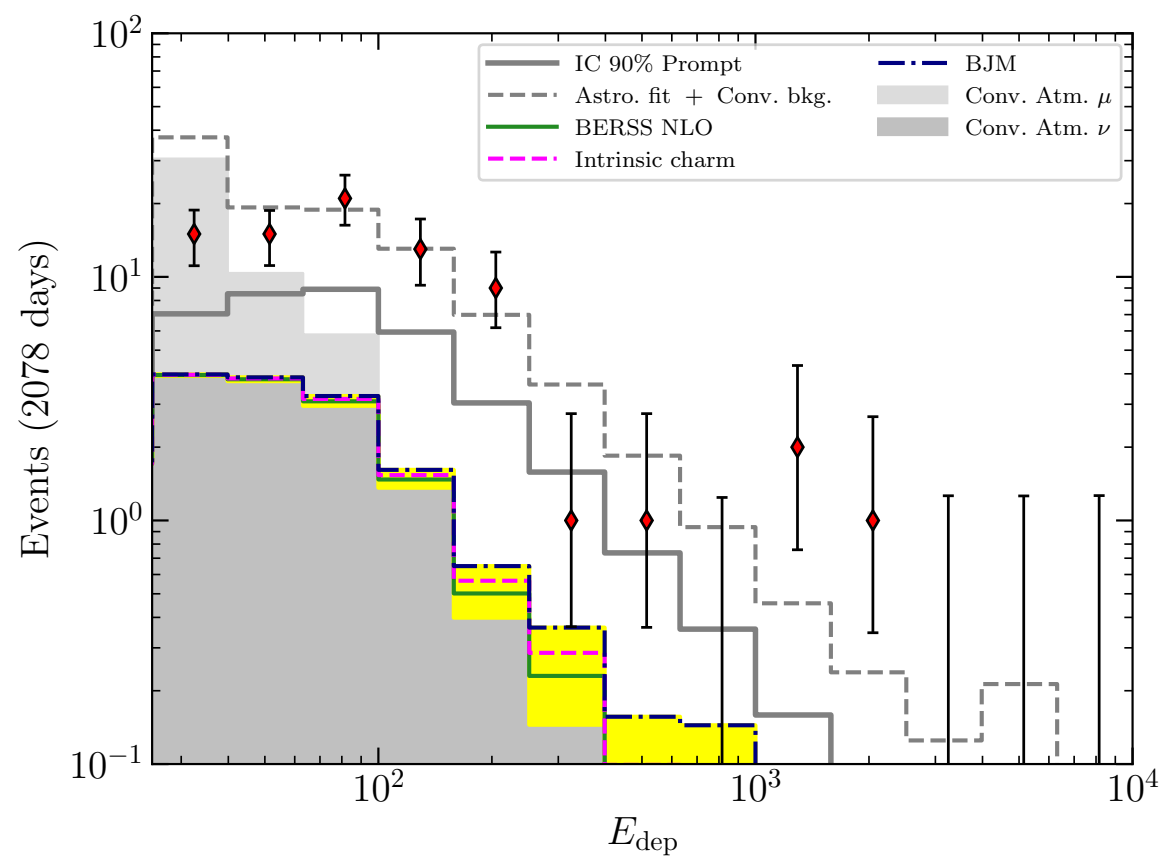

Figure 6. Event rates at IceCube showing the total atmospheric background (prompt + conventional) when different intrinsic charm model contributions are taken into account.

\section{Event rates}

IceCube has been steadily accumulating events over the last seven years. Its latest results [30] see 56 high-energy starting events within the energy range of $10 \mathrm{TeV}-2.1 \mathrm{PeV}$. By looking at the angular distribution of the incident background neutrinos, IceCube is capable of distinguishing between those from the prompt flux, which shows a largely flat distribution, and those from the conventional flux which is dominated by the vertical flux and suppressed toward the horizons. With present data, IceCube sees no evidence of the prompt flux yet, and accordingly sets a $90 \%$ confidence level upper bound at about 0.52 times the best-fit from [31]. The total prompt flux, even when including that from the BJM recombination and intrinsic charm, is consistent with this limit. Future analyses, involving more data and possibly improved by incorporating "self-veto" methods to distinguish between signal and background neutrinos [32], are expected to improve this limit.

The overall modification to the prompt atmospheric neutrino background in terms of IceCube 6-year event rates plotted against the energy $\left(E_{\text {dep }}\right)$ deposited in the detector, courtesy interactions of the lepton with detector nuclei, is shown in figure 6. While we have not re-evaluated the modification to the statistical significance of the non-atmospheric signal in light of the modified background, it is clear from looking at the figure that the change will be negligible. 


\section{Conclusions}

We have evaluated the upper limit to the contribution to prompt neutrino background from diffractive forward-rapidity cross sections by surveying existing models in the literature. As the rapidities where such contributions can be significant are limited in range, viz. at very high $x_{F}$, their contribution to the overall prompt-neutrino flux is limited to the very high energies at IceCube $E \gtrsim 200 \mathrm{TeV}$. As such, the background, even when accounting for novel diffractive production mechanisms, is a rather minor player in comparison to the flux of nonterrestrial neutrinos. We have evaluated the upper limit to this component, maintaining consistency with constraints at low and middle $x_{F}$ from accelerator experiments, and have estimated the expected event rates.

It is evident, from analyses at IceCube and ultra-high-energy cosmic-ray observatories, that an understanding of the origin of the extragalactic particles seen at energies of hundreds of $\mathrm{TeV}$ and higher requires quantitatively enhanced data, and more precision estimates of the various ingredients involved in their theoretical modelling. The background is an important part of this understanding.

We have shown here that there are large uncertainties in the QCD modelling of the prompt signal. However, in all considered cases, the contribution of novel mechanisms does not contribute significantly to the prompt neutrino signal.

\section{Acknowledgments}

We thank Grégory Soyez and Jean-Philippe Lansberg for useful discussions. We also thank the referee for helping us improve the discussions around our results. This work was supported by the Fonds de la Recherche Scientifique-FNRS, Belgium, under grant number 4.4501.15. AB is supported by the Fonds de la Recherche Scientifique de Belgique (F.R.S.FNRS) as "Chargé de Recherches" under grant number 1.B040.19. AB is also thankful to the computational resource provided by Consortium des É quipements de Calcul Intensif (CÉCI), funded by the F.R.S.-FNRS under grant number 2.5020.11 where a part of the computation was carried out.

Open Access. This article is distributed under the terms of the Creative Commons Attribution License (CC-BY 4.0), which permits any use, distribution and reproduction in any medium, provided the original author(s) and source are credited.

\section{References}

[1] B.P. Abbott et al., Multi-messenger Observations of a Binary Neutron Star Merger, Astrophys. J. 848 (2017) L12 [arXiv:1710.05833] [INSPIRE].

[2] M. Aartsen et al., Neutrino emission from the direction of the blazar txs 0506+056 prior to the icecube-170922a alert, Science 361 (2018) 147.

[3] Virgo, Ligo Scientific collaboration, B.P. Abbott et al., Observation of Gravitational Waves from a Binary Black Hole Merger, Phys. Rev. Lett. 116 (2016) 061102 [arXiv: 1602.03837] [INSPIRE]. 
[4] A. Bhattacharya, R. Gandhi and A. Gupta, The Direct Detection of Boosted Dark Matter at High Energies and PeV events at IceCube, JCAP 03 (2015) 027 [arXiv: 1407.3280] [INSPIRE].

[5] A. Bhattacharya, R. Gandhi, A. Gupta and S. Mukhopadhyay, Boosted Dark Matter and its implications for the features in IceCube HESE data, JCAP 05 (2017) 002 [arXiv: 1612.02834] [iNSPIRE].

[6] P. Nason, S. Dawson and R.K. Ellis, The Total Cross-Section for the Production of Heavy Quarks in Hadronic Collisions, Nucl. Phys. B 303 (1988) 607 [InSPIRE].

[7] P. Nason, S. Dawson and R.K. Ellis, The One Particle Inclusive Differential Cross-Section for Heavy Quark Production in Hadronic Collisions, Nucl. Phys. B 327 (1989) 49 [Erratum ibid. B 335 (1990) 260] [INSPIRE].

[8] A. Bhattacharya, R. Enberg, M.H. Reno, I. Sarcevic and A. Stasto, Perturbative charm production and the prompt atmospheric neutrino flux in light of RHIC and LHC, JHEP 06 (2015) 110 [arXiv: 1502.01076] [INSPIRE].

[9] A. Bhattacharya et al., Prompt atmospheric neutrino fluxes: perturbative QCD models and nuclear effects, JHEP 11 (2016) 167 [arXiv:1607.00193] [INSPIRE].

[10] LHCb collaboration, Measurements of prompt charm production cross-sections in $p p$ collisions at $\sqrt{s}=13 \mathrm{TeV}$, JHEP 03 (2016) 159 [Erratum ibid. 1609 (2016) 013] [arXiv: 1510.01707] [INSPIRE].

[11] S.J. Brodsky, P. Hoyer, C. Peterson and N. Sakai, The Intrinsic Charm of the Proton, Phys. Lett. B 93 (1980) 451.

[12] V.D. Barger, F. Halzen and W.-Y. Keung, The Central and Diffractive Components of Charm Production, Phys. Rev. D 25 (1982) 112 [INSPIRE].

[13] R. Vogt and S.J. Brodsky, QCD and intrinsic heavy quark predictions for leading charm and beauty hadroproduction, Nucl. Phys. B 438 (1995) 261 [hep-ph/9405236] [INSPIRE].

[14] E. Braaten, Y. Jia and T. Mehen, B production asymmetries in perturbative QCD, Phys. Rev. D 66 (2002) 034003 [hep-ph/0108201] [INSPIRE].

[15] E. Braaten, Y. Jia and T. Mehen, Charm anti-charm asymmetries in photoproduction from heavy quark recombination, Phys. Rev. D 66 (2002) 014003 [hep-ph/0111296] [INSPIRE].

[16] E. Braaten, Y. Jia and T. Mehen, The Leading particle effect from heavy quark recombination, Phys. Rev. Lett. 89 (2002) 122002 [hep-ph/0205149] [INSPIRE].

[17] E. Norrbin and T. Sjöstrand, Production mechanisms of charm hadrons in the string model, Phys. Lett. B 442 (1998) 407 [hep-ph/9809266] [INSPIRE].

[18] T.-J. Hou et al., CT14 Intrinsic Charm Parton Distribution Functions from CTEQ-TEA Global Analysis, JHEP 02 (2018) 059 [arXiv:1707.00657] [INSPIRE].

[19] B.A. Kniehl and G. Kramer, Charmed-hadron fragmentation functions from CERN LEP1 revisited, Phys. Rev. D 74 (2006) 037502 [hep-ph/0607306] [INSPIRE].

[20] J. Blümlein, A Kinematic Condition on Intrinsic Charm, Phys. Lett. B 753 (2016) 619 [arXiv: 1511.00229] [INSPIRE].

[21] R. Laha and S.J. Brodsky, IceCube can constrain the intrinsic charm of the proton, Phys. Rev. D 96 (2017) 123002 [arXiv: 1607. 08240] [INSPIRE]. 
[22] A.V. Giannini, V.P. Gonçalves and F.S. Navarra, Intrinsic charm contribution to the prompt atmospheric neutrino flux, Phys. Rev. D 98 (2018) 014012 [arXiv:1803.01728] [InSPIRE].

[23] W. Bai and M.H. Reno, Prompt neutrinos and intrinsic charm at SHiP, arXiv:1807.02746 [INSPIRE].

[24] F. Halzen and L. Wille, Charm contribution to the atmospheric neutrino flux, Phys. Rev. D 94 (2016) 014014 [arXiv: 1605.01409] [INSPIRE].

[25] F. Halzen and L. Wille, Upper Limit on Forward Charm Contribution to Atmospheric Neutrino Flux, arXiv: 1601.03044 [INSPIRE].

[26] W.K. Lai, A.K. Leibovich and A.A. Petrov, $D^{ \pm}$production asymmetry at the LHC from heavy quark recombination, Phys. Rev. D 90 (2014) 054022 [arXiv: 1408.2843] [INSPIRE].

[27] T.K. Gaisser, Spectrum of cosmic-ray nucleons, kaon production and the atmospheric muon charge ratio, Astropart. Phys. 35 (2012) 801 [arXiv:1111.6675] [INSPIRE].

[28] T. Gaisser, Cosmic rays and particle physics, Cambridge University Press, Cambridge U.K. (1990).

[29] P. Lipari, Lepton spectra in the earth's atmosphere, Astropart. Phys. 1 (1993) 195 [InSPIRE].

[30] IceCube collaboration, M.G. Aartsen et al., The IceCube Neutrino Observatory Contributions to ICRC 2015 Part II: Atmospheric and Astrophysical Diffuse Neutrino Searches of All Flavors, in Proceedings of 34th International Cosmic Ray Conference (ICRC 2015), The Hague, The Netherlands (2015) [arXiv: 1510.05223] [INSPIRE].

[31] R. Enberg, M.H. Reno and I. Sarcevic, Prompt neutrino fluxes from atmospheric charm, Phys. Rev. D 78 (2008) 043005 [arXiv: 0806. 0418] [InSPIRE].

[32] C.A. Argüelles, S. Palomares-Ruiz, A. Schneider, L. Wille and T. Yuan, Unified atmospheric neutrino passing fractions for large-scale neutrino telescopes, JCAP 07 (2018) 047 [arXiv:1805.11003] [INSPIRE]. 